\title{
TIPOS DE ESTRATÉGIAS UTILIZADAS PARA O ENSINO DA MEDIDA DA PRESSÃO ARTERIAL: REVISÃO INTEGRATIVA
}

\author{
TYPES OF STRATEGIES USED TO THE TEACHING \\ OF BLOOD PRESSURE MEASUREMENT: \\ INTEGRATIVE REVIEW
}

\author{
Bárbara Caroliny Pereiral \\ Rogério Silva Lima² \\ Silvana Maria Coelho Leite Fava ${ }^{3}$
}

\section{RESUMO}

Objetivo: identificar as evidências disponíveis na literatura sobre o ensino da medida da pressão arterial (PA) para os profissionais de saúde. Método: trata-se de uma revisão integrativa de estudos publicados no período de 2005 a 2015, no banco de dados da Coordenação de Aperfeiçoamento de Pessoal de Nível Superior, guiada pela metodologia de PICOD, a partir da pergunta norteadora: quais as evidências científicas na literatura sobre o ensino da medida da PA para profissionais de saúde? Resultados: dos seis artigos analisados, evidencia-se que cinco são internacionais, resultantes de estudos de baixos níveis de evidência. Constatou-se que a simulação tem se constituído uma estratégia metodológica predominante para o ensino da medida da PA. Conclusão: Há necessidade de desenvolvimento de novos modelos para o ensino da PA com incorporação de novas tecnologias, a partir de estudos longitudinais com fortes níveis de evidências para subsidiar práticas seguras.

Palavras-chave: Enfermagem. Conhecimento. Pressão arterial. Avaliação em enfermagem. Determinação da pressão arterial.

\section{ABSTRACT}

Objective: To identify the available evidences in the literature about the teaching of blood pressure (BP) measurement for health professionals. Method: Integrative review of studies published between 2005 and 2015 in the database of the Coordination of Improvement of Higher Level Personnel, guided by the methodology of PICOD, based on the guiding question: Which are the scientific evidences in the literature about the teaching of the BP measure for health professionals? Results: of the six articles analyzed, it was evidenced that five are international, resulting from studies of low levels of evidence. It was verified that the simulation has constituted a predominant methodological strategy for teaching the BP measure. Conclusion: There is a need for the development of new models for the teaching of BP with the incorporation of new technologies, from longitudinal studies with strong levels of evidence to subsidize safe practices.

Keywords: Nursing. Knowledge. Arterial Pressure. Nursing assessment. Blood pressure determination.

\section{INTRODUÇÃO}

A importância do conhecimento da técnica e da padronização da medida pressão arterial tem sido uma das metas da American Heart Association desde 1939, e tem suscitado discussóes sobre o

Enfermeira. Universidade Federal de Alfenas.

Enfermeiro. Mestre em Enfermagem. Universidade Federal de Alfenas.

Enfermeira. Doutora em Ciências. Universidade Federal de Alfenas. 
procedimento e proposto novas recomendaçóes e diretrizes sempre que avanços na área são descobertos ${ }^{(1)}$.

O Conselho Internacional de Enfermeiras tem reiterado que a medida da PA é a técnica mais exercida por enfermeiras em todo mundo, em todas as áreas da assistência, ou seja, primária, secundária ou terciária e mesmo com a evolução tecnológica não é uma técnica ultrapassada ${ }^{(2)}$.

A técnica da medida da pressão arterial se constitui em um processo simples, não invasivo, mas que se apresenta como um desafio, pois, para a aferição correta e obtenção de valores fidedignos que propiciem o acurado diagnóstico, é imprescindível o cumprimento dos procedimentos recomendados ${ }^{(3)}$. Por outro lado, quando realizada de forma incorreta pode levar a sérias consequências, como privar o cliente de um tratamento precoce da Hipertensão Arterial Sistêmica (HAS), leituras falsamente baixas, ou superestimar os valores da pressão levando a um tratamento desnecessário(3).

Portanto, para a medida da pressão arterial, deve-se levar em consideração, a técnica correta, os equipamentos adequados, as condiçóes de preparo do cliente para a medida e o método a ser utilizado ${ }^{(3)}$.

Em relação à técnica de aferição da $\mathrm{PA}$ com equipamentos aneroides, algumas medidas precisam ser seguidas para que se obtenha resultado fidedigno, tais como: o manguito deve ser colocado, sem folgas, 2 a $3 \mathrm{~cm}$ acima da fossa cubital, centralizado o meio de sua parte compressiva sobre a artéria braquial e o nível da pressão sistólica deve ser estimado pela palpação do pulso radial. A campânula ou o diafragma do estetoscópio deve ser colocado sem compressão excessiva, o esfigmomanômetro deve estar na altura dos olhos. Após esses cuidados, deve-se inflar rapidamente até ultrapassar em 20 a $30 \mathrm{mmHg}$ o nível estimado da pressão sistólica, obtido pela palpação, proceder à deflação lentamente, determinar a pressáo diastólica, auscultar cerca de 20 a $30 \mathrm{mmHg}$ abaixo do último som para confirmar o seu desaparecimento e depois proceder à deflação rápida e completa ${ }^{(1)}$.
Sempre levando em consideração a importância de explicar todo o procedimento para o cliente, principalmente para minimizar a ansiedade que pode aumentar os níveis da pressão sistólica.

A habilidade para a medida da PA está associada ao saber fazer, ou seja, à capacidade de executar e fazer uso produtivo do conhecimento adquirido com vistas à aferição de forma adequada. Muitas vezes, pela impossibilidade de avaliar adequadamente a habilidade dos estudantes de graduação em enfermagem em relação a medida da PA, há o comprometimento dos valores obtidos, interferindo na avaliação clínica e causando possíveis danos à pessoa. (4), Aponta, ainda, a escassez na literatura de instrumentos de avaliação, o que abre perspectiva para futuros estudos.

Os resultados encontrados na literatura, assim como em trabalho desenvolvido pela autora deste estudo, revelaram que ainda há erros na medida da pressão entre acadêmicos e profissionais de saúde, principalmente pela enfermagem ${ }^{(5-6)}$.

Em face desta realidade, torna-se necessário o desenvolvimento de estudos sobre metodologias mais eficazes para o ensino da medida da PA voltado para profissionais de saúde que possibilitem a criação de espaços de participação, reflexão e formaçãoo ${ }^{(3,7)}$.

A partir desta lacuna, e preocupados e comprometidos com a questão, buscou-se na literatura por melhores evidências para o ensino da medida da pressão arterial, com vistas à melhoria do processo ensino-aprendizagem, principalmente para a formação do enfermeiro, uma vez que é uma das técnicas mais realizadas no cotidiano da sua prática clínica. Diante ao exposto, o estudo teve por objetivo identificar as evidências disponíveis na literatura sobre o ensino da medida da pressão arterial para os profissionais de saúde.

\section{MÉTODO}

Trata-se de uma revisão integrativa da literatura que consiste em um método de pesquisa que proporciona uma investigaçáo que sintetiza e permite conclusóes gerais sobre determinada área 
de estudo, na qual um mesmo tema é abordado por diferentes estudos, sendo realizada de forma sistemática, com objetivo de contribuir para o conhecimento investigado e analisar conhecimentos pré-existentes ${ }^{(8)}$. Para o rigor metodológico do estudo, optou-se pelos critérios que compóem as seguintes etapas: 1) Identificação do problema e seleção da hipótese; 2) Estabelecimento de critérios de inclusão/exclusão de estudos; 3) Definição das informaçôes a serem extraídas dos estudos selecionados; 4) Análise das informaçóes; 5) Interpretação dos resultados e; 6) Apresentação da revisão/síntese do conhecimento ${ }^{(9-10)}$.

$\mathrm{Na}$ primeira etapa, para nortear a presente revisão, formulou-se a questão: quais as evidências científicas na literatura sobre o ensino da medida da pressão arterial para profissionais da saúde?, a partir da metodologia de PICOD. Nesse método, P corresponde a População (Participantes/ Estruturas): Profissionais de saúde; a letra I, intervenção (Relação de cuidado/ Processo): qual estratégia utilizada para ensino da medida da PA; letra C, Comparação das intervençôes: se existem diferentes estratégias para o ensino da medida da PA; letra $\mathrm{O}$, Resultados (Intermédios e finais): as estratégias mais eficazes para o ensino da medida da pressão arterial e; letra $\mathrm{D}$, Desenho do estudo: refere-se ao delineamento adotado pelo autor para desenvolvimento do estudo ${ }^{(11)}$.

Para os níveis de evidência, foram adotados os seguintes critérios: nível I: Revisōes sistemáticas ou metanálise de Estudo Clínico com randomização; nível II: Estudo Clínico com randomização; nível III: Estudo Clínico sem randomização; nível IV: Coorte e Caso controle; nível V: Revisão sistemática de estudos descritivos/ qualitativos; nível VI: Estudos descritivos/ qualitativos, nível VII: Opinião ${ }^{(12)}$.

$\mathrm{Na}$ segunda etapa, foram delimitados os seguintes critérios de inclusão: pesquisas publicadas em forma de artigo, em periódicos nacionais e internacionais, em português, inglês e espanhol; que apresentem estratégias para o ensino da medida da pressão arterial; publicadas no período de 2005 a 2015, com textos na íntegra. Foram excluídos as teses, livros, editoriais e estudos que abordam temas específicos como hipertensão arterial, fatores de risco, tratamento, conhecimento dos pacientes e de acadêmicos sobre HAS, monitorização residencial ou que não remetem ao tema.

$\mathrm{Na}$ sequência, foi realizada a busca da produção científica no período de agosto a setembro de 2015 nas bases e banco de dados: Literatura latino-americana e do Caribe em ciências da saúde (LILACS), Medical Literature Analysis and Retrieval Sistem (MEDLINE), Base de Dados de Enfermagem (BDENF), Cumulative Index to Nursing and Allied Health Literature (CINAHL), Base de dados Scientific Electronic Library online (SCIELO) enabiblioteca Cochrane, com o operador booleanos and, a partir do portal da Coordenação de Aperfeiçoamento de Pessoal de Nível Superior (CAPES), por meio dos seguintes descritores em Ciências da Saúde (DeCS) da Biblioteca Virtual de Saúde e descritores em língua inglesa Medical Subject Headings (MeSH): enfermagem, ensino, pressão arterial, conhecimento, avaliação em enfermagem, determinação da pressão arterial (nursing and teaching and arterial pressure and knowledge and nursing assessment and blood pressure determination).

A terceira etapa consistiu na definição das informaçôes a serem extraídas dos estudos selecionados que foram catalogados em uma planilha eletrônica e contemplou nesta etapa, a identificação do periódico, autores, idioma, objetivos, ano de publicação, local, delimitação da amostra, método, principais resultados, conclusôes, metodologias pedagógicas adotadas. $\mathrm{Na}$ quarta etapa, foi realizada a análise crítica dos artigos selecionados, o que permitiu buscar similitudes e diferenças. Após, foram catalogados de algo com suas semelhanças de estratégias utilizadas para o ensino da medida da PA.

A quinta etapa consistiu na interpretação dos resultados, na avaliação das metodologias utilizadas para ensino da medida da pressão arterial e das recomendações para prática, a partir das conclusōes. $\mathrm{Na}$ sexta, realizou-se a síntese do conhecimento das evidências sobre o ensino da medida da PA. Todas 
as estratégias foram descritas, com objetivos, sobre qual tipo de estratégia e suas conclusôes.

Os aspectos éticos foram adotados, dispensando a aprovação do Comitê de Ética. E não há conflito de interesse para realização desta revisão da literatura.

\section{RESULTADOS}

Foram encontrados 1598 artigos. Desses, 454 eram duplicados, resultando em 1144 artigos.
Após a primeira leitura do título e do resumo, foram selecionados 18 artigos e excluídos 1126, porque não correspondiam ao tema abordado. Os artigos foram lidos na íntegra e, de acordo com os critérios de inclusão, foram selecionados para esta revisão seis artigos. Doze artigos foram excluídos porque não respondiam à pergunta norteadora, relatavam sobre a hipertensão, medidas antropométricas, sedentarismo, conhecimentos dos pacientes sobre hipertensão arterial, tratamento com antihipertensivos, dentre outros.

Quadro 1- Distribuição das publicaçôes sobre as estratégias de ensino da medida da pressão arterial de 2006 - 2013.

\begin{tabular}{|c|c|c|c|}
\hline Ano & Título & Objetivos & Resultados \\
\hline 2006 & $\begin{array}{l}\text { O método auscultatório da pressão } \\
\text { arterial: nova abordagem para um } \\
\text { melhor ensino de cardiologia }\end{array}$ & $\begin{array}{l}\text { Este artigo propóe um novo sistema baseado } \\
\text { em um programa no computador para } \\
\text { melhorar a formaçáo clínica da } \\
\text { técnica auscultatória }\end{array}$ & $\begin{array}{c}\text { O sistema permite a verificaçáo se a PA foi } \\
\text { aferida de forma correta, permite a detecção } \\
\text { de comportamentos incorretos } \\
\text { no procedimento }\end{array}$ \\
\hline 2007 & $\begin{array}{l}\text { Aprendizagem baseado na simulação } \\
\text { para o ensino da medida da pressão } \\
\text { arterial entre estudantes de farmácia }\end{array}$ & $\begin{array}{l}\text { Avaliar o efeito da aprendizagem baseada } \\
\text { em simulação, capacidade dos alunos para } \\
\text { realizar avaliaçóes precisas da pressão arterial } \\
\text { e medir a satisfação do aluno com este novo } \\
\text { método de ensino }\end{array}$ & $\begin{array}{l}\text { Estudantes de farmácia mostraram melhora } \\
\text { significativa no desempenho de habilidades } \\
\text { clínicas e em seu conhecimento da } \\
\text { farmacoterapia da hipertensão }\end{array}$ \\
\hline 2011 & $\begin{array}{l}\text { Elaboração de uma hipermídia } \\
\text { educacional para o ensino do } \\
\text { procedimento de medida da } \\
\text { pressão arterial }\end{array}$ & $\begin{array}{c}\text { Construir uma hipermídia educacional para } \\
\text { o ensino da técnica de medida da } \\
\text { pressão arterial }\end{array}$ & $\begin{array}{l}\text { Além do Software que possibilita o aluno a } \\
\text { vivencias os passos da técnica da medida da } \\
\text { PA, utilizou-se exercícios e questionários para } \\
\text { intensificar a retençáo do conteúdo. }\end{array}$ \\
\hline 2012 & $\begin{array}{l}\text { Desenvolvimento e validação de } \\
\text { jogo educativo: medida da } \\
\text { pressão arterial }\end{array}$ & $\begin{array}{l}\text { Construir uma nova estratégia educativa } \\
\text { para ensino e fixaçăo da técnica da medida } \\
\text { da PA }\end{array}$ & $\begin{array}{l}\text { O jogo educativo despertou motivação } \\
\text { e curiosidade nos participantes, além do } \\
\text { interesse pelo aprendizado sobre a temática. }\end{array}$ \\
\hline 2013 & $\begin{array}{l}\text { Avaliaçáa da capacidade dos } \\
\text { estudantes de Farmácia para medir } \\
\text { com precisão Pressão Arterial } \\
\text { usando um braço simulador de } \\
\text { Pressão Arterial }\end{array}$ & $\begin{array}{l}\text { Comparar a precisão dos estudantes em } \\
\text { medir pressóes sanguíneas normais e elevadas } \\
\text { usando um braço simulador. }\end{array}$ & $\begin{array}{l}\text { Estudantes de farmácia podem precisar de } \\
\text { instruçóes adicionais e experiência com a } \\
\text { tomada de medidas de pressão arterial elevada } \\
\text { para garantir que eles são capazes de avaliar } \\
\text { com precisão este sinal vital importante }\end{array}$ \\
\hline 2013 & $\begin{array}{l}\text { A eficácia do treinamento sobre } \\
\text { Pressão Arterial baseada em } \\
\text { Simulação na pré-inscrição de } \\
\text { estudantes de Enfermagem }\end{array}$ & $\begin{array}{l}\text { Buscou-se avaliar a eficácia dos simuladores } \\
\text { de pacientes humanos na preparação de } \\
\text { estudantes de enfermagem para a medição } \\
\text { da pressão arterial na prática clínica. }\end{array}$ & $\begin{array}{l}\text { A precisão na tomada de PA não foi reforçada } \\
\text { pelo uso de um simulador de paciente, } \\
\text { apesar das melhorias em relaçáo à confiança e } \\
\text { competência técnica. }\end{array}$ \\
\hline
\end{tabular}

Fonte: Elaborada pelo Autor, 2018.

Dos seis artigos selecionados, quatro são de periódicos internacionais, sendo dois dos Estados Unidos (EUA), um da Itália e um da Austrália. O idioma inglês predominou em cinco publicações, seguido pelo português, em dois artigos. Constatou-se que, em sua maioria, o ensino da medida da PA teve como público alvo acadêmicos de enfermagem ou enfermeiros, seguido por acadêmicos de farmácia e de medicina. Com relação à formação dos autores, apenas dois artigos explicitaram a formação em enfermagem.
Os tipos de delineamentos dos estudos não se encontram explícitos nos artigos, mas de acordo com análise, quanto a classificação dos níveis de evidência, verificou-se que 50\% (3) com nível de evidencia VI, que corresponde a estudos descritivos/ qualitativos; $33,33 \%$ (2) com nível de evidência III, estudo clínico sem randomização e; $16,67 \%$ (1) nível IV, estudos Coorte e Caso controle ${ }^{(12)}$.

Os estudos selecionados foram classificados em três categorias de análise: 1) Estudos que 
utilizaram a simulação no ensino da medida da pressão arterial; 2) Estudos que utilizaram a tecnologia digital no ensino da medida da pressáo arterial e; 3) Estudo que utilizou a atividade lúdica no ensino da medida da pressão arterial.

\section{1) Estudos que utilizaram a simulação no ensino da medida da pressão arterial:}

A utilização de um braço simulador foi uma das estratégias utilizadas para o ensino da medida da PA. Previamente foi oferecida a orientação aos participantes sobre a técnica e os participantes tiveram a oportunidade de treinar um no outro, antes de realizar a técnica no braço simulador. Os autores sugerem a necessidade de mais estudos sobre estratégias de ensino ${ }^{(13)}$.

Em outro estudo que o simulador humano foi utilizado, os participantes receberam quatros horas de orientaçóes teóricas e práticas sobre a medida da PA, por meio de palestras, tutoriais e práticas em laboratório, com oportunidade de realizar o treinamento um no outro e com o simulador humano. Ao final, foi aplicado um teste sob a forma de questionário e de avaliação prática. A estratégia foi considerada positiva, no entanto, há a necessidade de aumentar a precisão e eficácia durante a medida ${ }^{(14)}$.

O simulador de um paciente a partir de um programa de computador permitiu que os manequins fossem controlados e programados para ter pulsos palpáveis, coração audível, pulmão e sons abdominais, parâmetros hemodinâmicos visíveis em um monitor e valores de pressão arterial. Era possível monitorar a taxa de deflação do manguito através de um gráfico de barras em tempo real e com isso dar um feedback para o participante. Essa estratégia de ensino resultou em satisfaçáo dos participantes, bem como o aumento da competência ${ }^{(15)}$.

\section{2) Estudos que utilizaram a tecnologia digital no ensino da medida da pressão arterial:}

A construção deumahipermídia educacional constituiu uma forma de utilizar a tecnologia como importante ferramenta e concluíram que o ambiente digital tem trazido efeitos positivos no processo de ensino aprendizagem. Constam no programa da hipermídia, os conhecimentos sobre anatomia, fisiologia, os métodos de medida da PA e a técnica de medida da PA e, ainda, uma relação de exercícios. As autoras ressaltaram a necessidade de mais estudos na área e a avaliação do impacto sobre o processo de ensino-aprendizado ${ }^{(3)}$.

Também foi desenvolvido um programa de computador que permitia gravar os valores da pressão e os sinais de áudio em tempo real durante a medida da PA. Primeiramente, cada participante teve a oportunidade de treinar a medida um no outro, antes de certificarem no programa. Esse método era capaz de identificar comportamentos errôneos durante a medida e fornecer um feedback positivo. Os autores concluíram que o programa foi de fácil execução e que pode auxiliar no ensino da medida da $\mathrm{PA}^{(16)}$.

\section{3) Estudo que utilizou a atividade lúdica no ensino da medida da pressão arterial:}

O jogo de dominó foi a estratégia de intervenção utilizada para ensino da medida da PA, construído com 28 peças, com imagens relacionadas à PA (alimentação não balanceada, consumo excessivo de sal, obesidade, tabagismo, etilismo, controle terapêutico medicamentoso, realização de exercício físico). Foi aplicado um questionário pré e pós-atividade educativa com questôes relacionadas ao tema pressáo arterial. Os autores sugerem a realizaçáo de mais estudos na área com a proposição de novas metodologias de ensino ${ }^{(7)}$.

\section{DISCUSSÃO}

A análise dos dados revela a escassez de estudos na temática, principalmente em âmbito nacional, e aponta como lacuna do conhecimento a ser explorada pelos pesquisadores interessados na temática. A escassez de trabalhos sobre o assunto pode ser atribuída ao fato de a temática não 
constituir uma prioridade de pesquisa ou uma preocupação destes profissionais em socializar as metodologias de ensino. Ademais, o fato de a formação dos autores não ter sido explicitada nos artigos, dificulta conhecer a produção científica dos diferentes profissionais de saúde sobre a temática.

Constatou-se, ainda, que a produção científica sobre o ensino da medida da PA em sua maioria decorre de estudos qualitativos que são classificados com evidências fracas, o que dificulta adotá-las na prática como melhores estratégias. A força da evidência está ligada ao nível de incerteza quanto à publicação, quanto ao efeito que se pode ter nos pacientes, e sobre quais intervençóes podem ser executadas. Portanto, quanto menor a força, maior o risco e efeitos causados nos pacientes ${ }^{(12)}$.

Em relação ao rigor da técnica, estudos têm demostrado que a prática nem sempre é realizada de forma correta pelos profissionais de saúde o que compromete a fidedignidade dos dados obtidos ${ }^{(17)}$. Os profissionais são suscetíveis a cometer eventos adversos com relaçáo aos processos técnicos e organizacionais e precisam trabalhar cada vez mais para minimizar os mesmos e proporcionar a segurança do paciente ${ }^{(17) .}$

Apreende-se que o processo de ensinoaprendizagem por meio da simulação tem sido uma estratégia predominante para o ensino da medida da pressão arterial e que tem revelado melhoria nas habilidades. Contudo, independente da estratégia, os autores têm recomendado que ela precisa ser testada e avaliada quanto a sua eficácia, quanto aos benefícios na prática clínica e quanto ao seu custo/benefício.

Defende-se, ainda, que a simulação desenvolve simultaneamente, aspectos cognitivos, psicomotores e afetivos e favorece o pensamento crítico, a capacidade de liderança, a tomada de decisão, a comunicação eficaz, a ética e a postura profissional, mas que todavia náo substitui a prática clínica $^{(18)}$.

As tecnologias são utilizadas de forma a transformar o que é complicado em útil, prática em dinâmica, além de ser mais criativo e estimulante. Servem como auxílio ao estudo e facilitam a aprendizagem, trazendo o conhecimento de forma mais estruturada. Assim como nos artigos selecionados, que usaram por meio de computadores e simuladores de forma a promover a aprendizagem $^{(19)}$.

Assim como o uso das tecnologias, as atividades lúdicas, mais especificamente os jogos, também podem auxiliar no processo de ensino-aprendizagem, fazendo com que os alunos se apropriem do conhecimento de forma lúdica, abrindo potencial para a criatividade e o raciocínio, além de melhor desenvolver aspectos sociais, pessoais e culturais ${ }^{(20)}$.

Neste sentido, há necessidade de repensar a prática pedagógica requerendo mudanças nos currículos, de forma a contemplar não só o interesse dos docentes, mas também o dos discentes que estão diretamente ligados ao processo de ensino-aprendizagem. A participação ativa destes pode motivá-los a pesquisar e buscar as melhores evidências para adotar sua prática clínica, tornando significativo o conhecimento e permitindo a construção de suas habilidades cognitivas ${ }^{(19)}$.

É imprescindível a formação de profissionais de saúde com capacidade crítica e com competência para vincular o conhecimento oriundo de pesquisas e de práticas clínicas para uma maior qualidade no atendimento. Os artigos analisados apontaram para a necessidade de novos estudos, principalmente, longitudinais e que avaliem a eficácia das estratégias quando adicionadas ao método de ensino.

\section{CONCLUSÃO}

Os dados resultantes desse estudo demonstram a escassez dos estudos desenvolvidos na temática em questão, principalmente nacionais. Evidencia-se que a simulação tem-se constituído como uma estratégia metodológica predominante entre os autores para o ensino da medida da PA e tem demonstrado melhoria na aquisição de habilidades. Há necessidade de avaliação das estratégias para aferir a sua eficácia. 
Este estudo traz contribuição para a ciência ao apontar a relevância da temática para a prática segura e ao compilar as diferentes estratégias adotadas pelos autores para o ensino da medida da pressão arterial.

Sugere-se o desenvolvimento de modelos para o ensino da PA com incorporação de novas tecnologias, a partir de estudos longitudinais com fortes níveis de evidências para subsidiar práticas seguras e a avaliação das estratégias para certificar a sua eficácia.

\section{REFERÊNCIAS}

1. Sociedade Brasileira de Cardiologia; Sociedade Brasileira de Hipertensão; Sociedade Brasileira de Nefrologia. VI Diretrizes Brasileiras de Hipertensão. Arq. Bras Cardiol. 2010; 95(1 supl.1):1-51.

2. Souza LP, Souza AAM, Silva JR. La hipertensión arterial: del conocimiento a la práctica de medir la presión arterial. Revista Digital. Buenos Aires. 2012. Enero. (164).

3. Alavarce DC, Pierin AMG. Elaboração de uma hipermídia educacional para o ensino do procedimento de medida da pressão artéria. Rev. esc. enferm. USP. 2011; 45(4): 939-44.

4. Tibúrcio MP, Melo GSM, Balduíno LSC, Costa IKF, Dias TYAF, Torres GV. Validação de instrumento para avaliação da habilidade de mensuração da pressão arterial. Rev Bras Enferm. 2014; 67(4): 518-7.

5. Ribeiro CCM, Lamas JLT. Comparação entre as técnicas de mensuração da pressão arterial em um e em dois tempos. Rev Bras Enferm. 2012; 65(4): 630-6.

6. Pereira BC. O ensino da medida da pressão arterial para profissionais da saúde: revisão integrativa [trabalho de conclusão de curso]. Alfenas: Universidade Federal de Alfenas; 2016.

7. Andrade LZC, Freitas DT, Holanda GF, Silva VM, Lopes MVO, Araújo TL. Desenvolvimento e validação de jogo educativo: medida da pressão arterial. Rev. enferm. UERJ. 2012; 20(3):323-7.

8. Souza MT, Silva MD, Carvalho R. Revisão integrativa: o que é e como fazer. Einstein. 2010; 8(1 Pt 1): 102-6.

9. Ganong LH. Integrative reviews of nursing research. Res Nurs Health. 1987; 10(1): 1-11.

10. Whittemore R, Knafl K. The integrative review: update methodology. J Adv Nurs. 2005; 52(5): 546-53.

11. Gião CR, Pinhão RC. Mapear o cuidado para regressar a casa. A Qualidade da Intervenção Educativa de Enfermagem no Planejamento da Alta da Pessoa Submetida a Transplante de Progenitores Hematopoiéticos [Dissertação de Mestrado]. Portugal: Instituto Politécnico de Setubal. Mestrado em Enfermagem Médico Cirúrgica, Escola Superior de Saúde; 2012.

12. Bernardo WM. Importância da análise dos níveis de evidência publicados. [Editorial]. Rev Assoc Med Bras. 2011; 57(1): 1.

13. Bottenberg MM, Bryant GA, Haack SL, North AM. Assessing Pharmacy Students' Ability to Accurately Measure Blood Pressure Using a Blood Pressure Simulator Arm. Am J Pharm Educ. 2013; 77(5): 1-5.

14. Gordon CJ, Frotjold A, Fethney J, Green J, Hardy J, Maw M, et al. The effectiveness of simulation-based blood pressure training in preregistration nursing students. Simul Healthc. 2013; 8(5): 335-40.

15. Seybert AL, Barton CM. Simulation-based learning to teach blood pressure assessment to doctor of pharmacy students. Am J Pharm Educ. 2007; 71(3): 1-6.

16. Corazza I, Fabbiani L, Marras L, Mariselli M, Marangoni F, Zannoli R. The Arterial Pressure Auscultatory Method: New Approach for Better Cardiology Teaching. High Blood Press Cardiovasc Prev. 2006 October; 13(4): 179-183.

17. Tibúrcio MP, Torres GV, Enders BC, Tourinho FSV, Melo GSM, Costa IKF. Análise contextual 
da mensuração da pressão arterial na prática clínica. J. res.: fundam. care. online. 2013; 5(3): 328-336.

18. Quilici AP, Abrão KC, Timermam S, Gutierrez F. Simulação clínica. Do conceito à aplicabilidade. São Paulo: Atheneu; 2012.

19. Souza IMA, Souza LVA. O uso da tecnologia como facilitadora da aprendizagem do aluno na escola. Itabaiana: Gepiadde. 2010; 8(4): 127-42.

20. Pinto IM, Botelho SSC. Ambientes Tecnológicos Lúdicos de Autoria (ATLA): criando espaços de ensino e aprendizagem. RBIE. 2012; 20(1): $133-45$.

Recebido em: 17/02/2018.

Aprovado em: 08/12/2018. 